\title{
A simple method of searching for a fixed solution in a coordinate domain
}

Sławomir Cellmer ( $\nabla$ slawomir.cellmer@uwm.edu.pl )

University of Warmia and Mazury

\section{Research Article}

Keywords: GNSS data processing, Ambiguity Function, integer least-squares, Voronoi cells, precise satellite positioning

Posted Date: January 31st, 2022

DOI: https://doi.org/10.21203/rs.3.rs-1301987/v1

License: (9) This work is licensed under a Creative Commons Attribution 4.0 International License. Read Full License 
1 A simple method of searching for a fixed solution in a coordinate domain.

2

3 Slawomir Cellmer*1, Krzysztof Nowel ${ }^{2}$, Artur Fischer ${ }^{3}$

$4 \quad *$ e-mail: slawomir.cellmer@uwm.edu.pl; ORCID: 0000-0002-2614-8017

$5 \quad{ }^{1,2,3}$ Department of Geodesy, Univ. of Warmia and Mazury, 1 Oczapowskiego Str., 10-719 Olsztyn, Poland

9 Abstract Recently, more and more Global Navigation Satellite Systems satellites are available for observation. Apart from obvious advantages, this brings new challenges in developing efficient computational methods of processing signals obtained from more satellites than to date. From the viewpoint of computation process efficiency, the most critical step is ambiguity resolution. Because of the discrete character of ambiguities, the search procedure is employed to perform this task. The search space dimension significantly impacts a search procedure computational load. The time needed for obtaining a solution raises considerably when the search space dimension is greater. Therefore, the improved version of the well-known concept of searching for a fixed solution in a three-dimensional coordinate domain was proposed and tested. The math model of the proposed approach is presented together with the algorithm of the search procedure. The numerical experiments were designed for simulated and real data. The simulated data has been prepared based on the concept proposed by the authors. This data simulation method is described in detail. The test results confirmed the reduction of the time needed to obtain the results when the proposed method is applied. This advantage over traditional methods unveils in the case of many satellites. Moreover, the real-data test pointed out that using the new approach is beneficial in reducing the computation time also in the case of a few satellites if long sessions are processed.

Keywords GNSS data processing · Ambiguity Function · integer least-squares · Voronoi cells $28 \cdot$ precise satellite positioning

\section{Introduction}

32 The Global Navigation Satellite Systems (GNSS) growth brings both new opportunities and challenges in signal processing methods. The apparent advantage of the increasing number of 
satellites possible to observe is the rise in the number of observations collected by the GNSS receiver. On the other hand, besides new signals, each new satellite provides new unknown values, i.e., ambiguities to be estimated. The rise in amounts of parameters substantially impacts the computational load. Even the most popular and efficient methods of optimizing the computational process in the classic approach, for example, in the LAMBDA method (Teunissen, 1995, 1999; de Jonge and Tiberius, 1996; Liu et al., 1999; Chang et al., 2005; Zhou 2011; Xu, 2012) are sensitive to the number of ambiguities. Xu proved that if observed satellites exceed a specific number, even the most efficient decorrelation techniques do not improve the search procedure efficiency $(\mathrm{Xu}, 2001)$. If there is more than a specific number of satellites, the decorrelation procedure takes more time than the search procedure performed without such a transformation. Thus, the cost of the enhancement technique outnumbers the benefits that are got from it! Therefore, it is justified to reconsider the concept of searching for integer ambiguities in a coordinate domain. The search space dimension is constant and amounts to three in such a case. Thus, the search space dimension does not depend on the number of satellites. Consequently, the computational load of a search procedure does not rise with increasing the number of satellites. It can even decrease if a search region is set correctly. Usually, the search region is formed based on the float position covariance matrix. In that case, this region shrinks with an increasing number of satellites because this typically improves satellites' configuration geometry and float solution precision. Then, fewer candidates are needed for carrying out the search procedure. The concept of searching for a fixed solution in the coordinate domain dates back to the early years of satellite navigation system development. Its classic form utilizes the Ambiguity Function (AF) method in the computation process. The AF method of processing the GPS carrier phase observations was the first time proposed by Counselman and Gourevitch (1981) and then by Remondi (1984). A substantial improvement of the AF method has been proposed by Cellmer et al. (2010). They developed the Modified Ambiguity Function Approach (MAFA). A description of the MAFA method can be found in, for example, (Cellmer 20122013 2015; Cellmer et al. 201020172018 2021; Kwasniak et al. 2016 2017; Baselga 2010; Nowel et al. 2018). Some researchers use the MAFA method foundations in recent research in airplane attitude determination (Wang et al. 2007; Wang et al. 2019; Wu et al. 2019, Zhao et al., 2022). Nevertheless, we do not use the functional model of the MAFA method in the present contribution. Unlike the MAFA, all formulas used in the search procedure proposed in this article are based solely on the float solution results. In contrast, the MAFA method requires the whole carrier phase data set for forming the observation equations when testing the candidates. Still, however, we take advantage of the other solutions used in the MAFA method: creating the search region in the coordinate domain and setting an optimal search step (Cellmer et al. 2018, 2021). The search region is in the form 
of an error ellipsoid of the approximate position, and the optimal search step's length derivation is based on a concept of the Voronoi cells (VC) and an actual satellites' configuration. According to Hassibi and Boyd (1998), the Voronoi cell of the point $\mathrm{X}_{0}$ in a lattice is the set of points in space closer to $\mathrm{X}_{0}$ than to any other point in the lattice. $\mathrm{Xu}$ (2006) used the VC to analyze an ambiguity resolution problem. In Teunissen's integer equivariant estimation theory, the VC is called an "in-pull region," where distance accounts for the non-Euclidean metric (Teunissen, 1999, 2003). Cellmer et al. (2021) used the VC in the meaning of a region containing candidates that give the same solution from the methods based on searching for a fixed solution in a coordinate domain. This meaning of the VC is assumed in our work. The search step's length resulting from the method proposed by Cellmer et al. (2021) is the longest distance between neighboring candidates that one at the same time guarantees we do not skip any VC included in the search region. In contrast to the MAFA method, the search procedure proposed in this contribution does not need the whole data set to test successive candidates. The search procedure is conducted based on approximate position coordinates and their covariance matrix. Both magnitudes are obtained from a float solution.

In the next section, the classic form of the mixed integer-real least squares estimation math model is introduced to familiarize readers with the notation used in this work. The third section is devoted to the main topic of this work, i.e., the search procedure in a coordinate domain (SinCD). Sole results from a float solution are used in this procedure as input. Two types of computational experiments are described in the fourth section. They are based on real and simulated data. The results of those experiments illustrate the effects of the proposed method. The conclusions are presented in the last section.

\section{The classic approach of precise positioning}

The math model of precise satellite positioning is (Hoffman-Wellenhof et al., 2008; Leick et al., 2015):

$$
\mathbf{y}=\mathbf{A a}+\mathbf{B b}+\mathbf{e}
$$

where $\mathbf{y}$ is the data vector, $\mathbf{a}$ is the integer ambiguity vector, $\mathbf{b}$ is the real parameter vector, $\mathbf{A}$ and $\mathbf{B}$ are the corresponding design matrices, and $\mathbf{e}$ is the noise vector. The $\mathbf{y}$ vector consists of double differenced (DD) carrier phase observations and/or code observations. The b vector consists of the baseline components. Sometimes it can also contain other parameters, e.g., atmospheric corrections. The classic approach of precise satellite positioning consists of the three stages: 
1) The least-squares (LS) solution in which the integer nature of ambiguities is discarded. This solution is called the "float solution."

105 2) The integer ambiguity resolution. This stage also includes a validation of the results.

106 3) The solution with integer ambiguities. This solution is called the "fixed solution."

107 The LS estimates of the $\mathbf{a}$ and $\mathbf{b}$ vectors, in the first stage, are obtained from:

$$
\left[\begin{array}{c}
\hat{\mathbf{a}} \\
\hat{\mathbf{b}}
\end{array}\right]=\left[\begin{array}{cc}
\mathbf{Q}_{\hat{\mathrm{a}}} & \mathbf{Q}_{\hat{\mathrm{a}} \hat{b}} \\
\mathbf{Q}_{\hat{\mathrm{b}} \hat{\mathrm{a}}} & \mathbf{Q}_{\hat{\mathrm{b}}}
\end{array}\right]\left[\begin{array}{l}
\mathbf{A}^{\mathrm{T}} \mathbf{P}_{\mathrm{y}} \mathbf{y} \\
\mathbf{B}^{\mathrm{T}} \mathbf{P}_{\mathrm{y}} \mathbf{y}
\end{array}\right]
$$

109 where: $\left[\begin{array}{ll}\mathbf{Q}_{\hat{a}} & \mathbf{Q}_{\hat{a} \hat{b}} \\ \mathbf{Q}_{\hat{b} \hat{a}} & \mathbf{Q}_{\hat{b}}\end{array}\right]=\left[\begin{array}{cc}\mathbf{A}^{\mathrm{T}} \mathbf{P}_{\mathrm{y}} \mathbf{A} & \mathbf{A}^{\mathrm{T}} \mathbf{P}_{\mathrm{y}} \mathbf{B} \\ \mathbf{B}^{\mathrm{T}} \mathbf{P}_{\mathrm{y}} \mathbf{A} & \mathbf{B}^{\mathrm{T}} \mathbf{P}_{\mathrm{y}} \mathbf{B}\end{array}\right]^{-1}$ and $\mathbf{P}_{\mathbf{y}}$ is the observation weight matrix.

110 The LS estimator of the integer ambiguity vector is defined as:

$$
\breve{\mathbf{a}}=\arg \min _{\mathbf{z} \in Z^{n}}\|\hat{\mathbf{a}}-\mathbf{z}\|_{\mathbf{Q}_{\hat{a}}}^{2}
$$

112 The expression at the $\arg \min ($.$) operator denotes a weighted norm of the ( \hat{\mathbf{a}}-\mathbf{z})$ vector:

$$
\|\hat{\mathbf{a}}-\mathbf{z}\|_{\mathbf{Q}_{\hat{a}}}^{2}=(\hat{\mathbf{a}}-\mathbf{z})^{\mathrm{T}} \mathbf{Q}_{\hat{\mathrm{a}}}^{-1}(\hat{\mathbf{a}}-\mathbf{z})
$$

114 It is necessary to employ the search procedure since the solution domain of formula (3) has a

115 discrete character. The ambiguity resolution step is always associated with the validation of the

116 results. The last step in precise positioning is a "fixed solution," which incorporates the integer

117 ambiguities obtained in the previous step:

$$
\breve{\mathbf{b}}=\hat{\mathbf{b}}-\mathbf{Q}_{\hat{b} \hat{a}} \mathbf{Q}_{\hat{a}}^{-1}(\hat{\mathbf{a}}-\breve{\mathbf{a}})
$$

119 The critical part of this three-step approach of precise positioning is the step of ambiguity

120 resolution. To date, many improvements to the search procedure have been developed and

121 successfully applied in practice. However, still, the search procedure is conducted in $n$-dim

122 ambiguity space. The concept of moving the search procedure from the $n$-dim ambiguity space

123 to the 3-dim coordinate space is presented in the next section.

\section{The search procedure in a coordinate domain}

126 The conditional solution (5) derived from model (2) describes the change of the b vector resulting from the modification of the a vector. The method proposed in this work utilizes the relationship of type (5) but with swapped the $\mathbf{a}$ and $\mathbf{b}$ vectors. This modification also concerns corresponding cofactor matrices. The conditional solution resulting from the model (2) can be 
alternatively formulated as the change of the a vector resulting from the change of the $\mathbf{b}$ vector.

Such a formula can be derived using block matrix:

$$
\mathbf{T}=\left[\begin{array}{ll}
\mathbf{I} & -\mathbf{Q}_{\hat{a} \mathrm{~b}} \mathbf{Q}_{\hat{b}}^{-1}
\end{array}\right]
$$

133

136

where the first block I is an identity matrix. Premultiplying normal equation system (2) by the $\mathbf{T}$ matrix, we get the formula for the â vector conditioned by the $\hat{\mathbf{b}}$ vector:

$$
\hat{\mathbf{a}}=\left(\mathbf{Q}_{\hat{\mathrm{a}}}-\mathbf{Q}_{\hat{\mathrm{a}} \hat{\mathrm{b}}} \mathbf{Q}_{\hat{\mathrm{b}}}^{-1} \mathbf{Q}_{\hat{\mathrm{b}} \mathrm{a}}\right) \mathbf{A}^{\mathrm{T}} \mathbf{P}_{\mathrm{y}} \mathbf{y}+\mathbf{Q}_{\hat{\mathrm{a}} \hat{\mathrm{b}}} \mathbf{Q}_{\hat{\mathrm{b}}}^{-1} \hat{\mathbf{b}}
$$

Thus the change in the $\mathbf{b}$ vector, such as, e.g., $\breve{\mathbf{b}}=\hat{\mathbf{b}}+\Delta \mathbf{b}$ results in the modification of the $\mathbf{a}$ vector:

$$
\breve{\mathbf{a}}=\left(\mathbf{Q}_{\hat{a}}-\mathbf{Q}_{\hat{a} \hat{b}} \mathbf{Q}_{\hat{b}}^{-1} \mathbf{Q}_{\hat{\mathrm{b}} \hat{a}}\right) \mathbf{A}^{\mathrm{T}} \mathbf{P}_{\mathrm{y}} \mathbf{y}+\mathbf{Q}_{\hat{\mathrm{a}} \mathrm{b}} \mathbf{Q}_{\hat{\mathrm{b}}}^{-1} \breve{\mathbf{b}}
$$

The difference of the equations (7) and (8) leads to:

$$
\breve{\mathbf{a}}=\hat{\mathbf{a}}-\mathbf{Q}_{\hat{\mathrm{a} b}} \mathbf{Q}_{\hat{\mathrm{b}}}^{-1}(\hat{\mathbf{b}}-\breve{\mathbf{b}})
$$

The description of applying the above formula in the proposed method is presented in the further part of this section. This method is carried out according to the following steps: forming a search region in coordinate space, subsequently establishing a grid of candidates inside it, then testing each candidate and choosing this, which best satisfies the criterion assumed on the tests' stage. Although the main stages of the proposed approach are similar to those in the classic approach, the details differ significantly. The proposed method assumes that the float solution is obtained the same way as the traditional approach. Next, the search region, as the confidence region of the float solution, is established. In the 3-dim coordinate space, this region takes the form of an error ellipsoid of an approximate position from the float solution. The initial candidates (IC) grid is formed inside the search space. The arrangement of the IC set has to guarantee that no the $\mathrm{VC}$ of integer ambiguity vector located inside the search region is omitted. On the other hand, to satisfy the computational efficiency, the distance between neighboring IC should be as long as possible so that the number of IC is small. The rules of forming the search region and setting the grid of IC inside it, which account above conditions, are described in detail by Cellmer et al. (2021). Figure 1 shows some examples of an arrangement of IC inside the error ellipsoid. The examples are taken from the tests described in the last section of the article. The essence of the proposed method is included in the next step of the data processing. Let us name the ICs with $\mathbf{b}_{\text {IC }}$. For $i$-th $\mathbf{b}_{\text {IC }}$ we compute the corresponding ambiguity vector:

$$
\mathbf{a}_{I C i}=\hat{\mathbf{a}}-\mathbf{Q}_{\hat{\mathrm{a}} \mathbf{b}} \mathbf{Q}_{\hat{\mathrm{b}}}^{-1}\left(\hat{\mathbf{b}}-\mathbf{b}_{I C i}\right)
$$



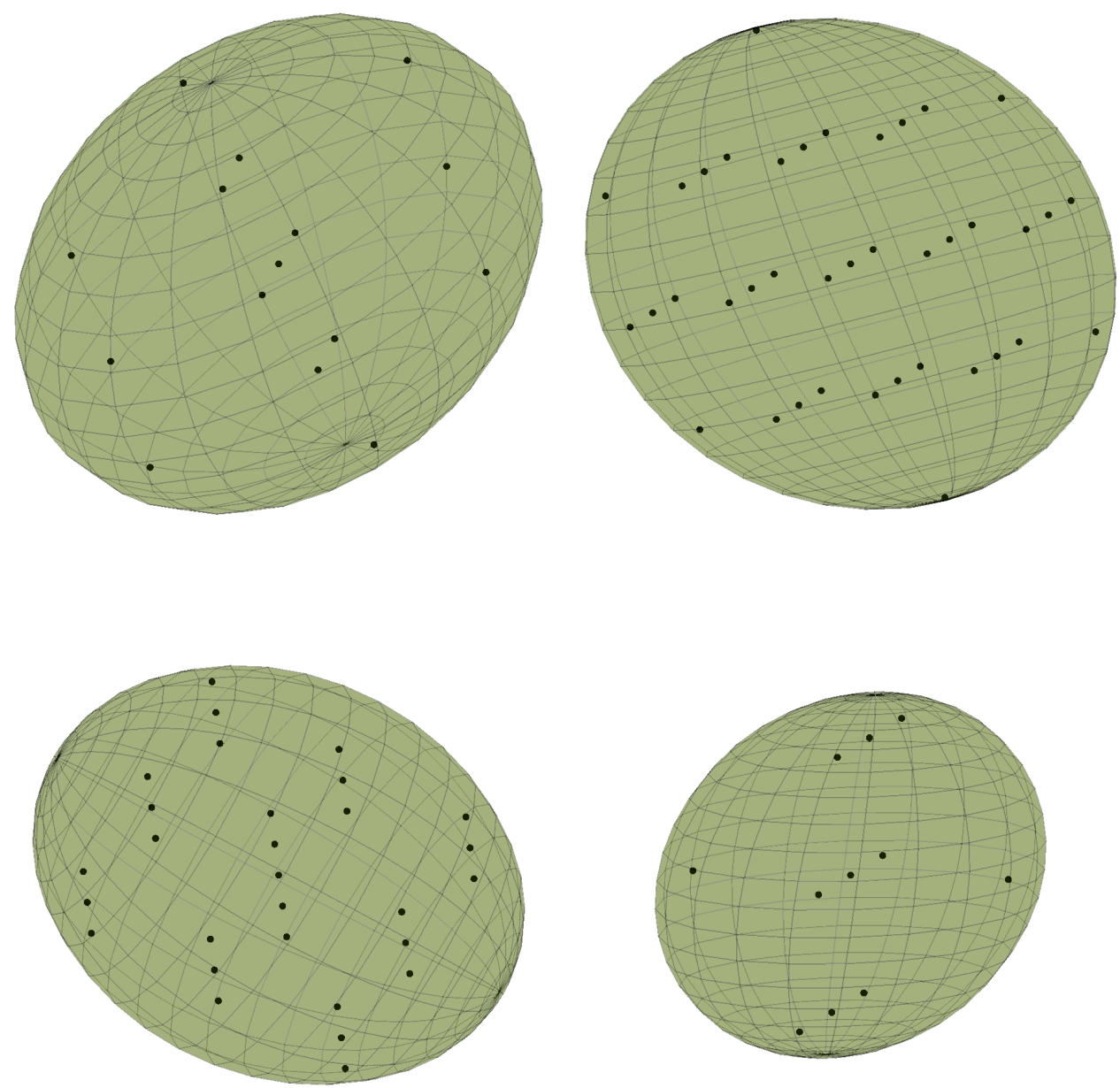

162 Fig. 1 Examples of the arrangements of the initial candidates inside the error ellipsoid.

163

164 Each ambiguity vector obtained by (10) is rounded to the nearest integer vector:

165

$$
\mathbf{a}_{F C i}=\operatorname{round}\left(\mathbf{a}_{I C i}\right)
$$

166 The integer vector $\mathbf{a}_{\mathrm{FCi}}$ corresponds to the $i$-th final candidate. Each vector $\mathbf{a}_{\mathrm{FC} i}$ is tested to find 167 that one which minimizes criterion:

168

$$
\mathbf{a}_{F C \min }=\arg \min _{\mathbf{z} \in Z^{n}}\left\|\hat{\mathbf{a}}-\mathbf{a}_{F C i}\right\|_{\mathbf{Q}_{\hat{a}}}^{2}
$$

169 The $\mathbf{a}_{\mathrm{FC} \text { min }}$ vector is employed to compute the final solution:

170

$$
\mathbf{b}_{f i x}=\hat{\mathbf{b}}-\mathbf{Q}_{\hat{b} \hat{a}} \mathbf{Q}_{\hat{a}}^{-1}\left(\hat{\mathbf{a}}-\mathbf{a}_{F C \min }\right)
$$


171 The successive steps of the computational process are depicted in Figure 2. The first three steps

172 of the computation process were discussed in (Cellmer et al., 2018; 2021). The primary issue

173 of the proposed method is included in the fourth step of the computational process: ICs testing.

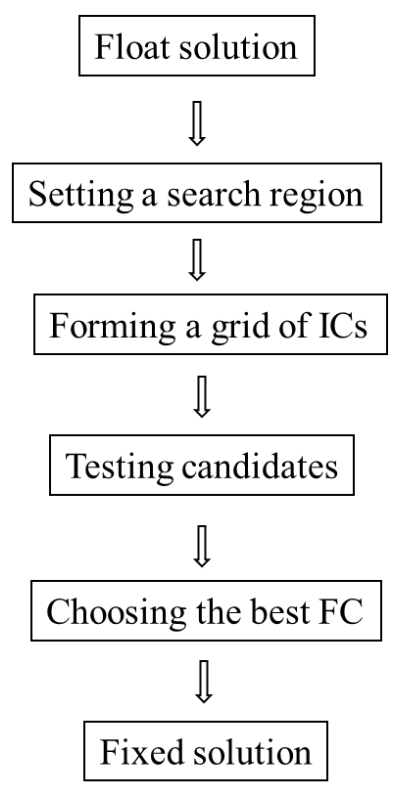

176 Fig. 2 Computational process of precise positioning based on search procedure in coordinate

177 domain

178 This step comprises operations (10) and (11), and also computing the norm on the right hand 179 of formula (12). Note the number of ICs can be greater than the number of FCs. The rules of 180 setting optimal search steps guarantee that no integer candidate is skipped. On the other hand, 181 a few ICs may fall into the same VC and consequently give the same FC. This is the cost of 182 reducing the dimension of a search space and has no significant impact on a computational load 183 if a search step length is set correctly.

\section{Numerical experiments}

186 The real data test

187 The first experiment is based on real data collected in two permanently operating EUREF 188 stations: WTZR and WTZZ (Germany), on 12, May, 2019, during a 24 h observation session. 189 The subject of the experiment is to test the performance of the new search procedure. The 190 analysis compares the new method with the classic one implemented in the LAMBDA method.

191 In the latter approach, a search for a fixed solution conducts in an ambiguity domain. The very 192 short baseline (1.62 m) was chosen for the tests to avoid any impact of atmospheric delays as extraneous factors irrelevant in comparative analysis. Such a short baseline lets to take the 
assumption that all atmospheric delays are eliminated by forming double differences of observations. Only the single-frequency GPS signals were processed in the experiment. The

196 data used in this test was already utilized in another experiment, described by Cellmer et al.

197 (2021). The interval between consecutive epochs was 30 seconds. The WTZR was set as a

198 reference station and the WTZZ as a rover. The precise position of the rover was computed

199 based on the data collected in a $24 \mathrm{~h}$ session. This position was used as a reference for further

200 comparisons. Next, the whole observation set was divided into short observation sessions. The

2012000 subsequent epochs at 30 -second intervals from the beginning of the $24 \mathrm{~h}$ observation

202 session were adopted as the starting epochs for the successive short sessions. The tests were

203 carried out for sessions of various lengths: from a single-epoch solution to 30 minutes sessions.

204 There were 2000 separate sessions tested for each session length. There were 7 satellites in

205 most sessions. Only in some cases were observations collected from 8 satellites. The approximate position in each session was obtained using the DD code and phase observations discarding the integer nature of ambiguities (the float solution). The error ellipsoid of the approximate position was formed using the covariance matrix from a float solution and setting the confidence level to 0.999 . The final precise position was estimated using two approaches.

210 The first way is the method proposed in the article and the second way is the LAMBDA 211 method. For simplicity, the first approach is denoted as \#1 and the second as \#2. The results

212 were compared. In the second approach, the computations were conducted using the Matlab 213 function LAMBDA_06.m retrieved from the TU Delft website:

214 https://www.tudelft.nl/citg/over-faculteit/afdelingen/geoscience-remote-

215 sensing/research/lambda/lambda (LAMBDA method, 2021). Table 1 lists the solutions' 216 success rates (SR) in both approaches. The SR values were computed separately for each 217 session length. The ambiguities obtained from $24 \mathrm{~h}$ session were assumed to be actual.

218 Table 1 Success rates as a percentage of correct solutions in the samples of 2000 observation sessions

\begin{tabular}{|c|c|c|c|c|c|c|c|c|c|}
\hline & \multicolumn{7}{|c|}{ Session length [minutes] } \\
\hline & $\begin{array}{c}\text { Single- } \\
\text { epoch }\end{array}$ & 1 & 2 & 5 & 10 & 15 & 20 & 30 \\
\hline & \multicolumn{8}{|c|}{ Success rates [\%] } \\
\hline \#1 (SinCD) & 45.6 & 59.8 & 70.2 & 85.3 & 94.8 & 98.2 & 98.6 & 100.0 \\
\hline$\# 2$ (LAMBDA) & 45.2 & 60.7 & 72.4 & 90.0 & 99.8 & 100.0 & 100.0 & 100.0 \\
\hline
\end{tabular}

220 Thus, the tested short-session solutions containing the actual ambiguity values are considered the correct solutions. The SR values are expressed in the percentage of correct solutions among 2000 sessions with a given length. The differences in SR ranged from 0 (for 30 min sessions) to 5 (for 10 min sessions). The proposed method achieved higher SR for single-epoch solutions.

224 In other cases, better SR was obtained by the LAMBDA method. Although the percentage of 
correct solutions is not precisely the same in both approaches, the differences in their values are minor. The mean value of absolute differences in SR in both approaches is $2.0 \%$. The core element of the analysis is a comparison of the time needed for finding the correct solution in both approaches. Figure 3 depicts the mean computation time computed from 2000 sessions separately for each session length. On the $\mathrm{X}$-axis are session lengths expressed in minutes, whereas on the $\mathrm{Y}$-axis is a computation time in seconds. The log scale has been applied on the 231 Y-axis. The session length has no impact on computation time in the LAMBDA method. Its 232 value amounts slightly over 10-3 seconds for all session lengths.

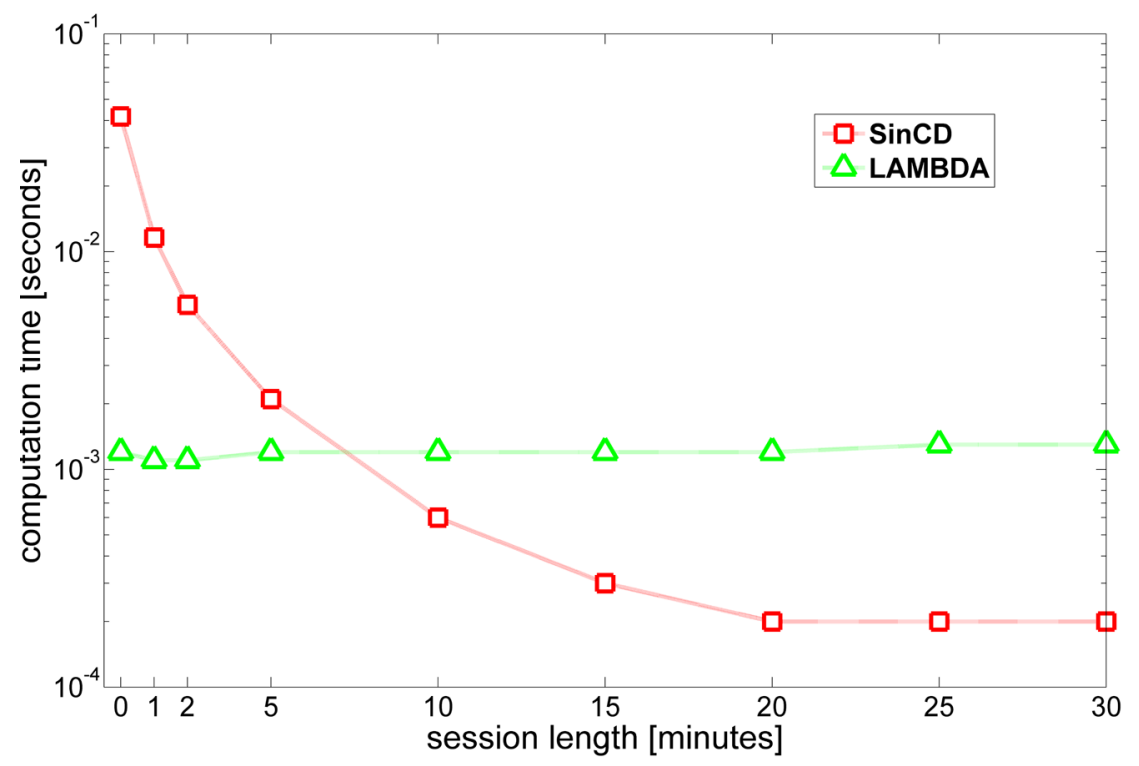

234 Fig. 3 Relationship between a session length and a computation time in the SinCD and the LAMBDA methods.

236 At the same time, the impact of session length on computation time is significant in the SinCD method. Its value ranges from $0.2 * 10-3$ (for the longest sessions) to $41.8 * 10-3$ in the case of single-epoch positioning. Shorter computation time in the SinCD method than in the LAMBDA is for 10-minute and longer sessions. In the present case study, the number of satellites amounts to 7 or, in some sparse sessions, 8. A relationship between the number of satellites and a computation time in both approaches can be evaluated based on simulated data. The following experiment has been designed for this purpose.

\section{The test based on simulated data}

245 It has been assumed that the positioning was conducted based on single-epoch data. The computations were repeated 2000 times for each assumed number of satellites. The satellite distribution was generated randomly in each epoch. 
The simulated code-observations set can be modeled using simulated observation residuals:

$$
\nabla \Delta \mathbf{P}=\nabla \Delta \boldsymbol{\rho}^{\text {(actual) }}+\nabla \Delta \mathbf{v}_{\mathrm{c}}
$$

250 or using simulated shift between approximated position and actual position

$$
\nabla \Delta \mathbf{P}=\nabla \Delta \boldsymbol{\rho}^{(\text {actual })}+\delta \boldsymbol{\rho}
$$

252

253

where: $\nabla \Delta \mathbf{P}$ is double differenced (DD) code-observations vector, $\nabla \Delta \mathbf{v}_{\mathrm{c}}$ is DD codeobservation residuals vector, $\nabla \Delta \boldsymbol{\rho}^{(\text {actual })}$ is DD actual geometric distances vector, $\delta \boldsymbol{\rho}$ is a part of a computed DD geometric distance, resulting from a shift of approximated position regard to the actual one. The last term in (11) can be expressed in linear form as:

$$
\delta \boldsymbol{\rho}=\mathbf{A d x}
$$

where the A matrix is a design matrix of DD code-observations.

Subtracting (15) from (14) and inserting (16) we get observation equations for simulated codedata:

$$
\nabla \Delta \mathbf{v}_{\mathrm{c}}=\mathbf{A d x}
$$

in which the $\nabla \Delta \boldsymbol{\rho}^{\text {(actual) }}$ and the $\nabla \Delta \mathbf{P}$ vectors vanish. All terms of (17) are prepared in the simulation procedure. Each entry of the $\nabla \Delta \mathbf{v}_{\mathrm{c}}$ vector on the left side of equation (17) is formed by generating four residuals with assumed normal distribution $N\left[0, \sigma_{c}\right]$ where $\sigma_{c}$ is the standard deviation of code-observations and then forming single and double differences. Four residuals have to be generated because this amount of pseudo-ranges is needed to create a single DD observation. The entries of the $\mathbf{d x}$ vector are values generated from the range of plus/minus a few meters. Preparing a design matrix A for simulated data set does not require knowledge about exact satellite positions. The $j$ th row of the undifferenced design matrix $\mathbf{A}_{\mathbf{u}}$ consist of the ratios of components of satellite-rover distance and this distance:

$$
\mathbf{A}_{\mathbf{u}}(j,:)=\left[-\frac{\Delta x_{r o v}^{j}}{\rho_{\text {rov }}^{j}},-\frac{\Delta y_{\text {rov }}^{j}}{\rho_{\text {rov }}^{j}},-\frac{\Delta z_{\text {rov }}^{j}}{\rho_{\text {rov }}^{j}}\right]
$$

That vector can be alternatively expressed by azimuth $A z_{j}$ and elevation angle $E l_{j}$ in a local, horizontal coordinate system:

$$
\mathbf{A}_{\mathbf{u}}(j,:)=\left[-\cos \left(E l_{j}\right) \cos \left(A z_{j}\right),-\cos \left(E l_{j}\right) \sin \left(A z_{j}\right),-\sin \left(E l_{j}\right)\right]
$$


Thus, it is enough to generate satellites' azimuths and elevation angles to form a design matrix

275

276

277

278

279

280

Figure 4 depicts the computation time (CT) needed to obtain a solution by two tested methods. for undifferenced observations. The A matrix for DD observations is created by subtracting the row related to the reference satellite from the other rows of the $\mathbf{A}_{\mathbf{u}}$ matrix:

$$
\mathbf{A}(j,:)=\mathbf{A}_{\mathbf{u}}(j,:)-\mathbf{A}_{\mathbf{u}}(r e f,:), \text { for all } j \neq r e f .
$$

Thus, a design matrix for simulated data is formed using satellites' azimuths and elevations generated in ranges $(0 ; 2 \pi)$ and $(0 ; \pi / 2)$ respectively. The observation equations for the simulated carrier phase observations can be derived analogously. They take the form similar to those of the equation (17):

$$
\nabla \Delta \mathbf{v}_{\mathrm{ph}}=\frac{1}{\lambda} \mathbf{A d x}+\mathbf{a}
$$

The only difference is an additional term of the ambiguity vector a, which must be generated as a random integer number. The DD carrier phase residuals on the left hand are expressed in cycles, so the first term on the right hand is divided by carrier phase wavelength $\lambda$. There were considered the various number of satellites in a single-epoch positioning. The computations were repeated 2000 times for each number of satellites. Data were simulated and processed separately in each epoch using two approaches: the SinCD and the LAMBDA methods. The single-epoch positioning was conducted in a two-stage process: In the first stage, the observation set consisted of both types of code- and phase observations. The float solution results, not satisfying the integer nature of ambiguities, were the same in the two approaches. The precise position (fixed solution) was computed using the SinCD and LAMBDA methods. The SRs calculated from 2000 repetitions achieve almost the same values in both approaches for all tested numbers of satellites. The only difference in those values occurs in the case of 9 satellites and amounts to $0.1 \%$ (99.9\% in the SinCD method and $100 \%$ in the LAMBDA method). Table 2 lists the SR values for both methods with respect to the number of satellites. Since the SR for the number of satellites in the range of 10-30 achieves the same values amounting to $100.0 \%$ they have been placed in the single column.

Table 2 Success rates as a percentage of correct solutions in 2000 repetitions of single-epoch positioning for various numbers of satellites

\begin{tabular}{|c|c|c|c|c|c|}
\hline & \multicolumn{5}{|c|}{ Number of satellites } \\
\hline & 6 & 7 & 8 & 9 & $10-30$ \\
\hline & \multicolumn{5}{|c|}{ Success rates [\%] } \\
\hline \#1 (SinCD) & 37.4 & 84.4 & 99.0 & 99.9 & 100.0 \\
\hline \#2 (LAMBDA) & 37.4 & 84.4 & 99.0 & 100.0 & 100.0 \\
\hline
\end{tabular}
The CT was computed as a mean value from 2000 repetitions for each of the assumed numbers 
of satellites. The CT of the LAMBDA method for the number of satellites fewer than 10 is stable and low. In contrast, the computational time of the SinCD method significantly decreases, in this range, when the number of satellites increases. The opposite situation starts when the number of satellites amounts to 20 or more. The computational time of the LAMBDA method starts to increase considerably, whereas the computational time of the SinCD method decreases gradually. Starting from 25 satellites providing the observations for a single-epoch positioning, the computational time of processing data is shorter in the SinCD method than in the LAMBDA method.

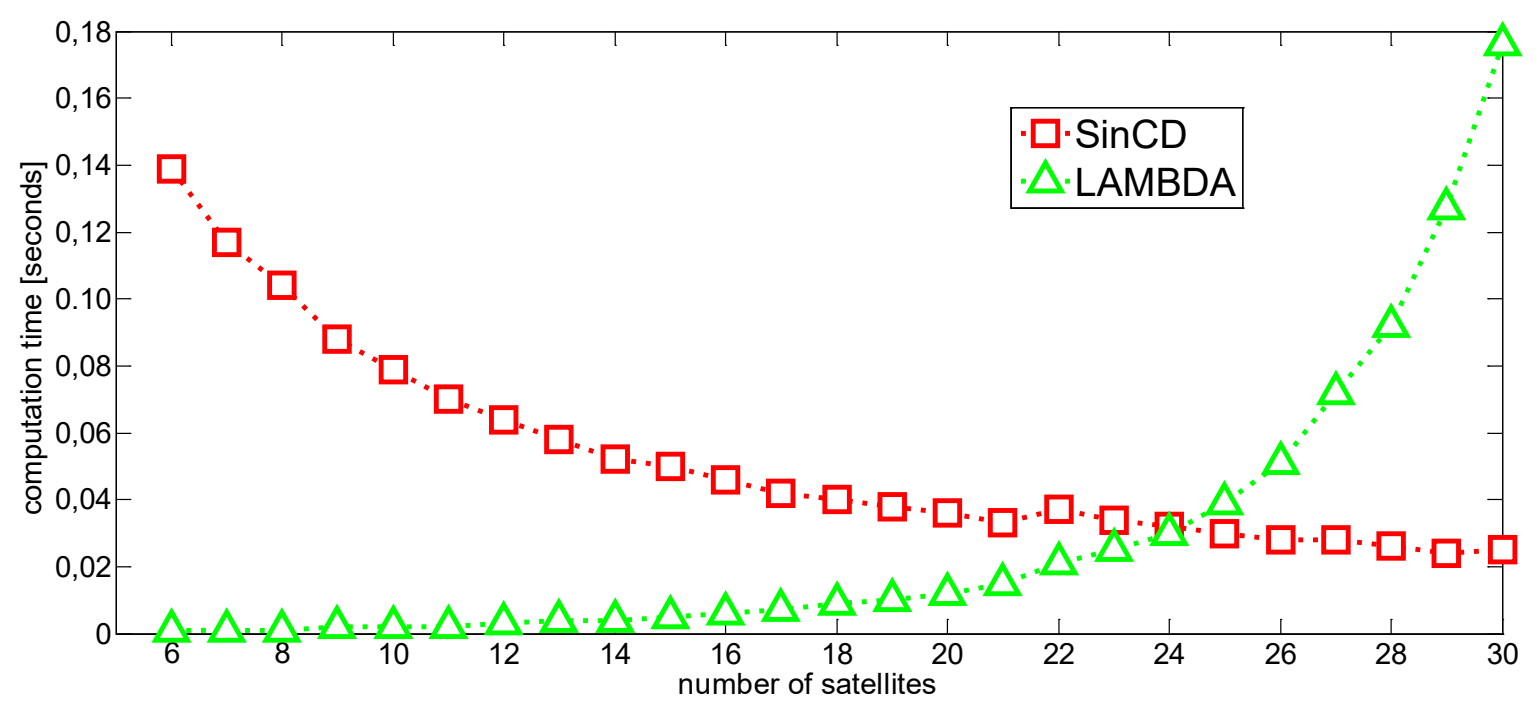

313 Fig. 4 The computation time of the SinCD and the LAMBDA methods for a various number 314 of satellites. The single-epoch positioning was performed based on simulated data.

316 The results of the tests confirmed the good performance of the proposed method.

317 Unfortunately, based on the real data test, we should admit that the SR is slightly fewer than in 318 the traditional method in the cases with a small number of satellites and short observation 319 sessions. However, the difference is not significant. On the other hand, for sessions 10-minute 320 and longer with 7 or 8 satellites providing observation set, the CT is shorter in the proposed 321 method than in the traditional one. Based on the test with simulated data, one can conclude that 322 in a case of a single-epoch positioning, the CT is shorter in the proposed method than in the 323 traditional one if the number of satellites is greater than 24 .

\section{Conclusions}

326 The motivation of the work was the apparent finding that the dimension of the search space is 327 non-negligible for search procedure efficiency. The time needed for obtaining a solution raises 328 considerably when the search space dimension is greater. Therefore, the concept of searching 
for a fixed solution in a coordinate domain instead of in an ambiguity domain was investigated.

330 As a result, the new method was developed and tested. A major advantage of the proposed method is moving a search procedure from multi-dimensional ambiguity space to only threedimensional coordinate space. Such an operation allows shortening computation time, thereby increasing the efficiency of the precise positioning. This feature reveals oneself in the case of an observation set obtained from many satellites. It has great importance in the prospect of increasing the number of satellites resulting from developing modern satellite navigation systems. The test results confirmed shortening computation time when using the proposed method and that its advantage over traditional methods unveils in the case of many satellites. The real data test pointed out that applying the new approach is beneficial in the case of long sessions, even if we deal with a small number of satellites.

Acknowledgments This research was supported by the National Science Centre, Poland, grant number 2018/31/B/ST10/00262.

\section{References}

Baselga S. (2010) Global optimization applied to GPS positioning by ambiguity functions. Meas Sci Technol 21(12):125102, DOI: http://dx.doi.org/10.1088/0957-0233/21/12/125102

Cellmer S (2012) A graphic representation of the necessary condition for the MAFA method.

Cellmer S (2013) Search procedure for improving modified Ambiguity Function Approach. Surv Rev 45(332):380-385

Cellmer S (2015) Validation procedure in the Modified Ambiguity Function Approach. Geodynamica et Geomaterialia 12(178):135-144

Cellmer S, Wielgosz P, Rzepecka Z (2010) Modified Ambiguity Function Approach for GPS carrier phase positioning. J Geodesy 84(4):264-275

Cellmer S, Nowel K, Kwasniak D (2017) Optimization of a grid of candidates in the search procedure of the MAFA method. In: Environmental Engineering 10th International Conference, April 27-28 2017, Vilnius, Lithuania

Cellmer S, Nowel K, Kwaniak D (2018) The new search method in precise GNSS positioning. IEEE T Aero Elec Sys 54(1):404- 415 
Cellmer S. Nowel K, Fischer A. (2021) A search step optimization in an ambiguity functionbased GNSS precise positioning. Surv Rev, DOI: 10.1080/00396265.2021.1885947

362

Chang X, Yang X, Zho T (2005) MLAMBDA: a modified LAMBDA method for integer least squares estimation. J Geodesy 79(9):552-565

Counselman C, Gourevitch S (1981) Miniature interferometer terminals for earth surveying: ambiguity and multipath with the global positioning system. IEEE T Geosci Remote 19(4):244-252

de Jonge PJ, Tiberius CCJM (1996) The LAMBDA method for integer ambiguity estimation: implementation aspects. Publications of the Delft Computing Centre, LGR-Series No. 12

Hassibi A, Boyd S (1998) Integer parameter estimation in linear models with applications to GPS. IEEE T Signal Proces 46(11):2938-2952

Hofmann-Wellenhof B., Lichtenegger H., Wasle E. (2008) GNSS-Global Navigation Satellite Systems - GPS, GLONASS, Galileo \& more. Springer-Verlag, Vienna, 2008.

Kwasniak D, Cellmer S, Nowel K (2016) Schreiber's differencing scheme applied to carrier phase observations in the MAFA method. In: Proceedings of 2016 Baltic Geodetic Congress (BGC Geomatics), pp. 197-204

Kwasniak D, Cellmer S, Nowel K (2017) Single frequency RTK positioning using Schreiber's differencing scheme. In: 2017 Baltic Geodetic Congress (BGC Geomatics), Gdansk, Poland, pp. $307-311$

LAMBDA method from TU Delft (Accessed May 9, 2021, at https://www.tudelft.nl/citg/overfaculteit/afdelingen/geoscience-remote-sensing/research/lambda/lambda)

Leick, A., Rapoport, L., Tatarnikov, D. (2015) GPS Satellite Surveying (4 ${ }^{\text {th }}$ edn). John Wiley and Sons, Hoboken, New Jersey.

Liu LT, Hsu HT, Zhu YZ, Ou JK (1999) A new approach to GPS ambiguity decorrelation J Geodesy 73:478-490

Nowel K, Cellmer S, Kwasniak D (2018) Mixed integer-real least squares estimation for 386 precise GNSS positioning using a Modified Ambiguity Function Approach. GPS solut

Remondi B (1984) Using the Global Positioning System (GPS) phase observable for relative geodesy: modeling, processing and results. PhD thesis, Center for Space Research, University of Texas at Austin 
Teunissen P (1995) The least-squares ambiguity decorrelation adjustment: a method for fast GPS integer ambiguity estimation. J Geodesy 70(1-2):65-82

393 Teunissen, P. (1999) An optimality property of the integer least-squares estimator. J Geodesy 73, 587-593 https://doi.org/10.1007/s001900050269

Teunissen, P. (2003) Theory of integer equivariant estimation with application to GNSS. J Geodesy 77, 402-410. https://doi.org/10.1007/s00190-003-0344-3

Wang Y, Zhan X, Zhang Y (2007) Improved ambiguity function method based on analytical resolution for GPS attitude determination. Meas Sci Technol 18(9):2985-2990

Wang Y, Zhao X, Pang C, Wang X, Wu S, Zhang C (2019) Improved pitch-constrained ambiguity function method for integer ambiguity resolution in BDS/MIMU-integrated attitude determination. J Geodesy 93(4):56-572, DOI 10.1007/s00190-018-1182-7

Wu H, Zhao X, Pang C, Zhang L, Feng B (2019) Multivariate constrained GNSS real-time full attitude determination based on attitude domain search. J Navigation 72(2):483-502, DOI $10.1017 / \mathrm{S} 0373463318000784$

Xu P (2001) Random simulation and GPS decorrelation J Geodesy 75(7-8): 408-423

Xu P (2006) Voronoi cells, probabilistic bounds, and hypothesis testing in mixed integer linear models. IEEE T Inform Theory 52(7):3122-3138

Xu P (2012) Parallel Cholesky-based reduction for the weighted integer least squares problem J Geodesy 86(1):35-52

Zhou Y (2011) A new practical approach to GNSS high-dimensional ambiguity decorrelation

412 Zhao Y, Zou J, Zhang P, Guo J, Wang X, Huang G. (2022) An Optimization Method of 413 Ambiguity Function Based on Multi-Antenna Constrained and Application in Vehicle 414 Attitude Determination. Micromachines; 13(1):64. https://doi.org/10.3390/mi13010064

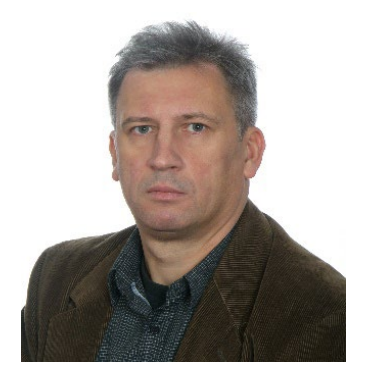

SLAWOMIR CELLMER received the Ph.D. degree in geodesy in 2002 from the University of Warmia and Mazury in Olsztyn, Poland. In 2013 he received habilitation (postdoctoral degree). He is an Associate Professor at the Faculty of Geoengineering at the University of Warmia and Mazury in Olsztyn (UWM), Poland. His research interests cover computational methods and GNSS data processing. 


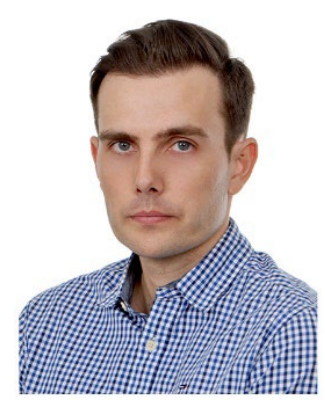

416

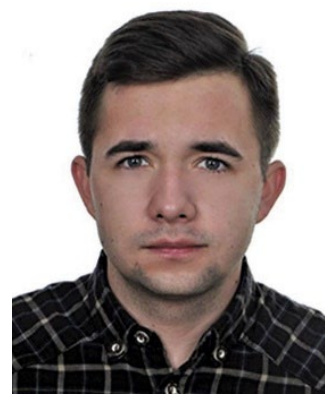

KRZYSZTOF NOWEL is an Assistant Professor at the Faculty of Geoengineering at the University of Warmia and Mazury in Olsztyn (UWM), Poland, where he earned his Ph.D. in Geodesy in 2015. His research interests include deformation measurement analysis and GNSS integer ambiguity resolution.

ARTUR FISCHER is a Ph.D. candidate at the Faculty of Geoengineering at the University of Warmia and Mazury in Olsztyn (UWM), Poland. His research interests contain computational methods in GNSS and engineering surveys. 